\title{
Unidades Productivas Integrales, como un producto Turístico para el Desarrollo Rural Sustentable
}

\author{
Unit's productive integrals, as a product of tourism for \\ sustainable Rural Development
}

\author{
Sonia Lucía Montero-Herrera ${ }^{1}$ \\ Universidad Nacional, Costa Rica
}

\begin{abstract}
Resumen
Las Unidades Productivas Integrales (UPI), son un sistema de producción que permite el uso óptimo de los recursos naturales y culturales, por medio del manejo de tecnologías de bajo costo, que promueven un desarrollo endógeno y auto sostenible y alta diversidad de productos agropecuarios, forestales y energéticos de origen local o adaptados al territorio.

Se caracterizan por ocupar mano de obra familiar, productos orgánicos de origen autóctono, comercializables y encadenables al producto turístico local. Su importancia, radica en que proporcionan, seguridad alimentaria, manejo integrado de los recursos, protección del medio ambiente, formación de microempresas y organización comunitaria. Por esta razón la Universidad Nacional de Costa Rica (UNA) con sus proyectos de extensión universitaria, acompañan a estas comunidades indígenas en la implementación de estas unidades. Como proyecto denominado: Autogestión y sostenibilidad de las unidades productivas en territorios indígenas del Valle la Estrella, con miras a la consolidación de un sistema de producción basado en una cosmovisión indígena e intercambio de saberes ancestrales en el Territorio Indígena Tayni, en el Valle de la Estrella, Limón, Costa Rica; el cual con técnicas participativas que tienden a favorecer el intercambio de saberes ancestrales como instrumento de aprendizaje. En este territorio la UNA ha logrado replicar el modelo de las UPI, en todos los centros educativos en torno a la primera iniciativa creada en el 2015, permitiendo la diversificación de los productos alimentarios en comedores estudiantiles locales y en la comunidad y en general, el rescate de productos medicinales tradicionales del bosque, y de la cultura local. El
\end{abstract}

1 Geógrafa, Master en Gestión de Turismo de Naturaleza de la Escuela de Ciencias Geográficas de la Universidad Nacional de Costa Rica, académica del Campus Sarapiquí, Universidad Nacional, Costa Rica, Correo electrónico: luciamonteroherrera@gmail.com.

Este artículo corresponde a la ponencia presentada en el 35th Conference of Latin American Geographers realizada en San José, Costa Rica del 20 al 22 de mayo del 2018 
proyecto ha generado empoderamiento local y ha creado la necesidad de incursionar en iniciativas de turismo rural comunitario, enfocado en los saberes ancestrales locales. Asociado con la motivación de los estudiantes de los centros educativos para ingresar en un futuro a la UNA, donde actualmente hay cinco estudiantes de este territorio de los cuales cuatro ya están por graduarse en las diferentes carreras del campus Sarapiquí.

Palabras clave: Unidades Productivas Integrales, Comunidades Indígenas y Turismo Rural Comunitario.

\begin{abstract}
The productive integrals units (UPI), are a production system that allows optimal use of natural and cultural resources through the management of low-cost technologies, promoting endogenous development and sustainable auto and high diversity of products agricultural, forestry and energy of local origin or adapted to the territory. They typically deal with family labor, organic products of indigenous origin, marketable and linkable to the local tourism product. Its importance lies in that provide food security, integrated management of resources, protection of the environment, formation of micro-enterprises and community organization. For this reason the National University of Costa Rica (UNA) with his extracurricular projects, accompany these indigenous communities in the implementation of these units. As a project called: self-management and sustainability of the productive units in indigenous territories of the Valley star, with a view to the consolidation of a system of production based on an indigenous cosmovision and ancestral knowledge exchange in the Territory Tayni indigenous, in the Valle de la Estrella, Limon, Costa Rica; which with participatory techniques which tend to favour the exchange of ancestral knowledge as a tool for learning. In this territory the one has managed to replicate the model of the UPI, in all schools around the first initiative created in 2015, allowing diversification of food products in local student canteens and in the community and in overall, the rescue of traditional medicinal products of the forest, and the local culture. The project has generated local empowerment and has created the need to venture into rural community tourism, focused on the local ancestral knowledge initiatives. Associated with the motivation of students in schools to enter in a future one, where there are currently five students of this territory, of which four are already graduating in the different careers of Sarapiquí Campus.
\end{abstract}

Keywords: comprehensive productive units, indigenous communities and community-based rural tourism

\title{
Introducción
}

La Universidad Nacional de Costa Rica conjuntamente con la Vicerrectoría de Extensión con el programa de Regionalización Universitaria perteneciente al Consejo Nacional de Rectores (CONARE), ha venido trabajando en los últimos años con las comunidades con mayores necesidades de desarrollo del país y dando prioridad a aquellas iniciativas que aborden entre sus objetivos la sustentabilidad. Tal es el caso del proyecto "Autogestión y sostenibilidad de las unidades productivas en territorios indígenas del Valle La Estrella, con miras a la consolidación de un sistema de producción basado en una cosmovisión indígena e intercambio de saberes ancestrales", cuyo enfoque es proporcionar seguridad alimentaria, manejo 
integrado de los recursos, protección del medio ambiente, organización comunitaria, formación de microempresas y la posible comercialización y encadenamientos con productos turísticos locales.

Las UPI como práctica agro-ecoturística novedosa utiliza técnicas que optimizan no solo la producción agropecuaria, el territorio tecnologías de bajo costo, sino también el uso sostenible de los recursos naturales como el bosque las plantas medicinales y de otros usos como la confección de ropa y productos autóctonos combinado al recurso humano y cultural que incluye la mano de obra familiar unida a los saberes y conocimientos ancestrales del territorio indígena donde se implementan las unidades productivas integrales

La Universidad Nacional y el territorio indígena Cábecar del Valle La Estrella, por medio de la utilización de técnicas participativas, dio inicio a este proyecto en el año 2015 en el Centro Educativo de segunda enseñanza de Boca Cohen, con el objetivo principal de insertar una UPI, diversificar la alimentación de los estudiantes en el comedor institucional, aprovechar el espacio físico y proyectarse como modelo a seguir para las instituciones educativas adyacentes y para la población en general, motivando el interés no solo por esta nueva técnica de producción sino también por involucrarse en otro sector del mercado como lo es el turismo étnico local.

\section{Antecedentes}

La comunidad indígenas Cábecar del Valle La Estrella está ubicada en la provincia de Limón, forma parte de los distritos con mayor pobreza con un porcentaje importante de población indígena tiene un acceso limitado, donde la fuente principal de empleo es la producción y comercialización de banano y plátano por parte de empresas transnacionales, que limita el ingreso económico en las familias que componen este territorio ubicándolos dentro del rubro de las regiones más rezagadas del país y en donde encontramos centros de poblados importantes como Boca Cohen, Gavilán, Jabuy, Vesta, Moi, Bellavista perteneciente al distrito del Valle La Estrella y la comunidad de Los Ángeles Cábecar ubicada en el sector suroeste del Valle colindante con el cantón de Talamanca (ver figura 1) 


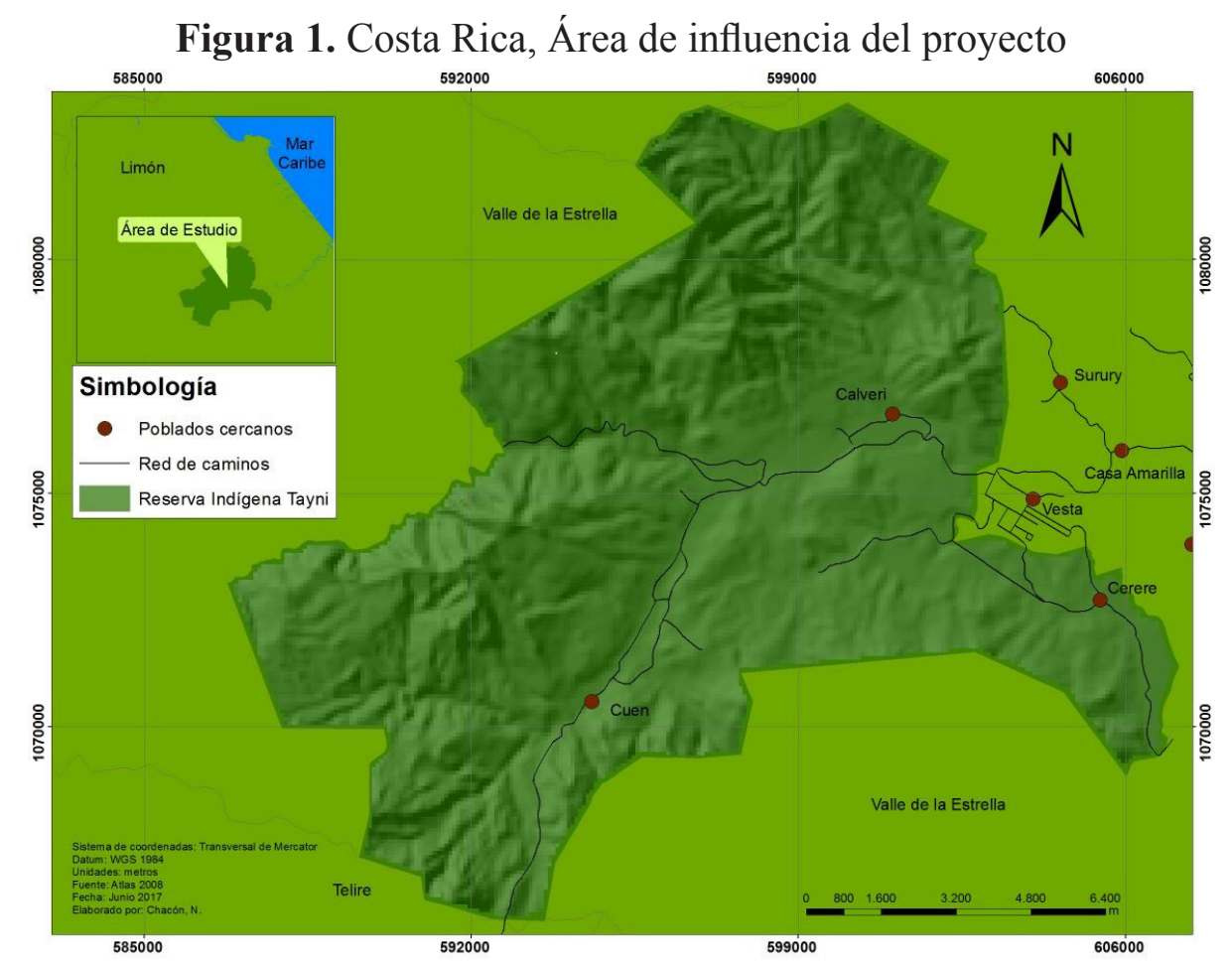

Fuente: Atlas 2008, elaborado por Chacón, N

Dado que la producción agrícola de Costa Rica todavía sigue la tendencia de la llamada "revolución Verde" que implica una agricultura extensiva e intensiva con monocultivos permanentes, en donde las técnicas agrícolas empleadas implican gran cantidad de uso del suelo, uso indiscriminado de plaguicidas y fertilizantes, tecnologías como el uso de maquinarias que arrasan con la biodiversidad local, que generan altos ingresos pero que no se ven reflejados en la comunidad, abaratando los costos de la mano de obra y deteriorando cada día más el medio ambiente. Se ha visto la necesidad de implementar otro tipo de producción más amigable con el ambiente de menor costo que implique menor uso de recursos con el suelo y que sobre todo sea diversa permitiendo optimizar la producción y el recurso con el que se cuenta.

Es a raíz de esta situación que se crea este proyecto con el fin de canalizar las necesidades detectadas, procurando mejorar las condiciones actuales por medio de técnicas novedosas como son las UPI involucrando a los actores locales y su cultura, saberes ancestrales y su cosmovisión 
Antes que nada se debe dar una breve reseña de lo que es la agricultura tradicional en Costa Rica, que no ha variado mucho desde las últimas décadas " sustentado por un interés de crecimiento económico de corto plazo, sin mediar el costo ambiental o social, tal es el caso del cultivo de piña, en el cual participan agricultores con poco conocimiento ambiental, escasos recursos económicos o tecnología, que en mayor o menor medida, contribuyen con el deterioro de la biodiversidad"(Estado de la Nación, 2008, pág. 3)

Las Unidades productivas integrales o UPI que es una opción sustentable para los agricultores son "... una alternativa de producción familiar integrada, basada en la implementación de sistemas autosuficientes y diversificados que minimiza de esta forma perdidas y desequilibrios en el ambiente.” (Rojas y Daly 2016, pág. 147)

Las Unidades Productivas Integrales (UPI) están inmersas en la nueva tendencia de la agricultura sostenible, basada en sistemas de producción que mejoran la calidad ambiental al tener menor impacto, mejoran los recursos básicos satisfaciendo las necesidades de materia prima que son económicamente más rentable por que la producción es más armoniosa y no requiere del factor químico como el uso indiscriminado de los plaguicidas que mejoran a su vez las condiciones de los agricultores.

La UPI que no solo aprovechan todo el espacio físico posible y que no necesariamente son grandes extensiones de territorio favorecen la pluralidad y calidad de la producción sino que pueden asegurar una mayor competitividad, el mantenimiento de las familias y el ingreso de los productores, y que en el fondo de su esencia complementa mucho la producción tradicional campesina y que adquiere en el caso de este proyecto el incentivo del conocimiento ancestral de actores indígenas que componen este proyecto.

Otro factor importante a considerar dentro de la UPI es la utilización de insumos propios, y recursos naturales adyacentes de manera eficiente y con muy poco impacto, mezclando lo natural con tecnologías propias del sector, sino que también puede permitir el ingreso al sector comercio con iniciativas de turismo o la venta de servicios como es la capacitación a través de la practica en el campo de este tipo de producción diversificada utilizándola como una aula abierta y participativa para todo aquel que esté interesado en generar este tipo de emprendimiento. 
El modelo de la UPI calza de manera completa con los objetivos del proyecto en las cuales las prácticas de conservación y uso sostenible de la biodiversidad en contraste con los sistemas tradicionales indígenas permiten aprovechar los recursos internos de la finca de forma sostenible con el uso de tecnologías de bajo costo y fácil adopción que a su vez permite desarrollar y fortalecer el conocimiento y habilidades de los grupos participantes y darle valor a la mano de obra familiar.

Dentro del término del desarrollo sostenible dado por Gómez. C, UNESCO (2017) “es aquel desarrollo que asegura satisfacer las necesidades del presente sin comprometer la capacidad de las futuras generaciones para satisfacer las propias." (Pág. 91). Donde la conservación de los recursos naturales debe jugar un papel importante en todos los sistemas de producción tanto agrícola, pecuaria y forestal y en donde el trabajo multidisciplinario es vital para el buen desempeño de este tipo de iniciativas por medio de la creación de servicios eco sistémicos como son la siembra y protección de árboles y el bosque, la extracción sostenible de las plantas útiles del bosque, la fijación de carbono, la conservación del recurso suelo mejoramiento y protección del recurso hídrico entre otros y que permita que las familias participantes se involucren dentro de otras actividades lucrativas como es el agro-eco-turismo.

Según la Organización Mundial de Turismo (OMT), (2018) "El turismo es un fenómeno social, cultural y económico relacionado con el movimiento de las personas a lugares que se encuentran fuera de su lugar de residencia habitual por motivos personales o de negocios/profesionales. Estas personas se denominan visitantes (que pueden ser turistas o excursionistas residentes o no residentes) y el turismo tiene que ver con sus actividades, de las cuales algunas implican un gasto turístico. (Pag.1)

En el caso del turismo para Costa Rica se viene desarrollando de manera eficaz desde hace varias décadas, con una marca establecida para el país que es reconocida a nivel mundial y en donde el crecimiento ha venido en aumento desde la década de los 80 generando directa e indirectamente aumento en las divisas y las actividades económicas de los lugares de visitación.

La relación de las UPI con el turismo está dada por medio de las nuevas tendencias del turismo que involucra al turismo étnico que es el preferido de las personas que gustan conocer otras culturas enriqueciendo 
las suya propia y recorriendo los diferentes lugares y culturas que hay el por el mundo. Uniendo lo anterior con el turismo sostenible que según la OMT (2018) es "El turismo que tiene plenamente en cuenta las repercusiones actuales y futuras, económicas, sociales y medioambientales para satisfacer las necesidades de los visitantes, de la industria, del entorno y de las comunidades anfitrionas "(p.1) Gracias a la diversidad de relaciones implicadas en la actividad turística, el turismo sostenible tiene la capacidad de actuar como catalizador de cambio en el mundo, beneficiando a la lucha de causas como el hambre, la paz y seguridad, el fomento de las economías locales.

Puede fusionarse todo esto con el agroturismo impulsador del desarrollo en zonas rurales y en donde las UPI apuntan a ser un modelo educativo y replicable que permite no solo la producción sostenible y la comercialización de los productos sino también presentarse a sí mismo como un modelo para el desarrollo en las comunidades rurales.

\section{Materiales y métodos}

Para dar inicio en la implementación de las UPI en este territorio indígena se decidió utilizar el enfoque acción-participación cuyo fin es solventar las necesidades identificadas, que en este caso es proponer un sistema alternativo que solucione las carencias de empleo, ingresos económicos, seguridad alimentaria y educación ambiental entre otros, reforzado con un aprendizaje mutuo basado en el respeto de los saberes ancestrales indígenas resaltando todas las fortalezas que esta cosmovisión posee. Para la puesta en práctica de esta iniciativa se cuenta con un equipo multisciplinario compuesto por académicos de la Universidad Nacional y maestros de las instituciones educativas con actores locales como son los dirigentes comunales y personas con amplios conocimientos en las cosmovisión indígena

Todo este proyecto se dio primeramente con diferentes pasos que son:

- $\quad$ Paso 1: Visitas de Campo: Estas incluyeron las primeras visitas de reconocimiento del territorio y la escogencia del lugar donde se iban a implementar las UPI

- $\quad$ Paso 2 Diagnostico: Este paso permitió identificar dentro del lugar escogido a aquellos actores locales, informantes claves, dirigentes comunales, las debilidades y fortalezas y los recursos propios, es 
aquí donde se identifican las partes más vulnerables y con mayor necesidad sin intentar imponer ninguna técnica ni resultado sino más bien promoviendo una participación conjunta de acuerdo con sus saberes ancestrales.

- Paso 3. Definición de la metodología, tomando en cuenta las características de la población meta, se optó por la metodología acción participación tanto en las instituciones públicas donde se desarrollan las UPI como en la comunidad que se integró al proyecto tiempo después, creando planes de trabajo con una visión conjunta aplicando tecnologías modernas como culturales y ancestrales propias de cada comunidad-

- Paso5: Talleres este paso es de gran importancia no solo por las capacitaciones y aprendizaje mutua, sino porque estos permitieron la inserción de otras escuelas y de familias vinculadas a estas instituciones de poder manifestar un interés en participar del proyecto como tal. También se procura en cada uno de los talleres que se realizan crear una conciencia ambiental por medio de la sensibilización de los participantes y buscando un empoderamiento de los mismos ya que ellos con su participación se convierten en los protagonistas principales del evento.

- $\quad$ Paso 6 Auto sostenibilidad y autogestión Este es el último paso en el que se pretende que cada institución y actor participante sean capaces de establecer metas propias auto gestionables, que sean capaces de empoderarse y adquirir compromisos no solo para la comunidad sino con el ambiente en procura de buscar un mejor desarrollo sostenible para su territorio.

\section{Resultados}

En el periodo de ejecución que ha tenido este proyecto de extensión universitaria en Territorio del Valle de La Estrella, podemos resaltar los siguientes resultados:

- Año 1 El establecimiento de la primera UPI ubicada en el Colegio Indígena Boca Cohen, que sirvió de modelo para que las otras instituciones se interesaran y se involucraran al año siguiente por iniciativa propia al proyecto. 
Sonia Lucía Montero-Herrera. Unidades Productivas Integrales, como un producto Turístico para el Desarrollo Rural Sustentable

Ilustración 1. Cama de hortalizas en la UPI del Colegio Indígena Boca Cohen, Territorio Indígena Tayni, Valle La Estrella, Limón Costa Rica, 2017

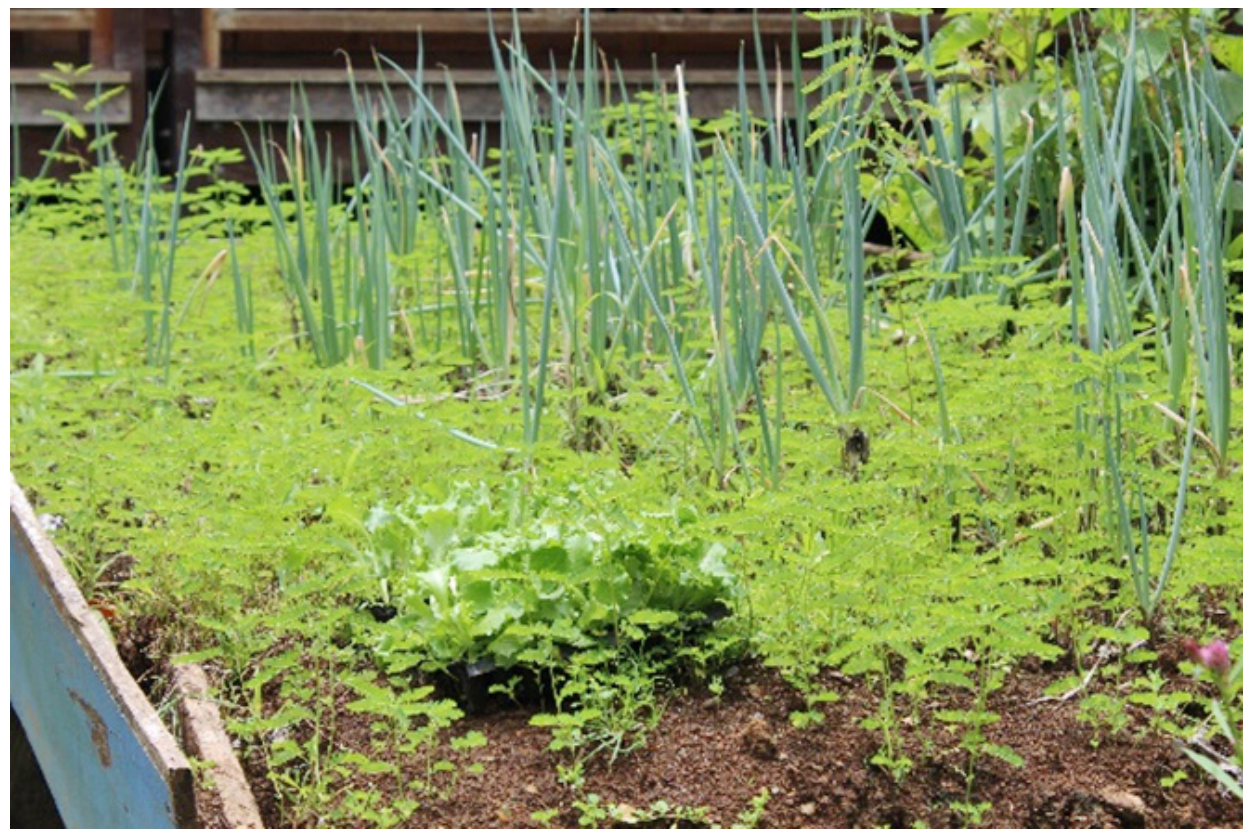

Fuente: Propia

- $\quad$ Año 2 Integración de cuatro instituciones educativas más al proyecto como fueron las Escuela Indígenas de Boca Cohen, Gavilán, Jabuy y las escuela de Vesta quienes por medio de la participación en los talleres realizados en el colegio externaron un interés en integrarse y poder beneficiarse de esta iniciativa. 
Ilustración 2. Cama de hortalizas en el la Escuela Indígena Jabuy, Territorio Indígena Tayni, Valle La Estrella limón Costa Rica. 2018

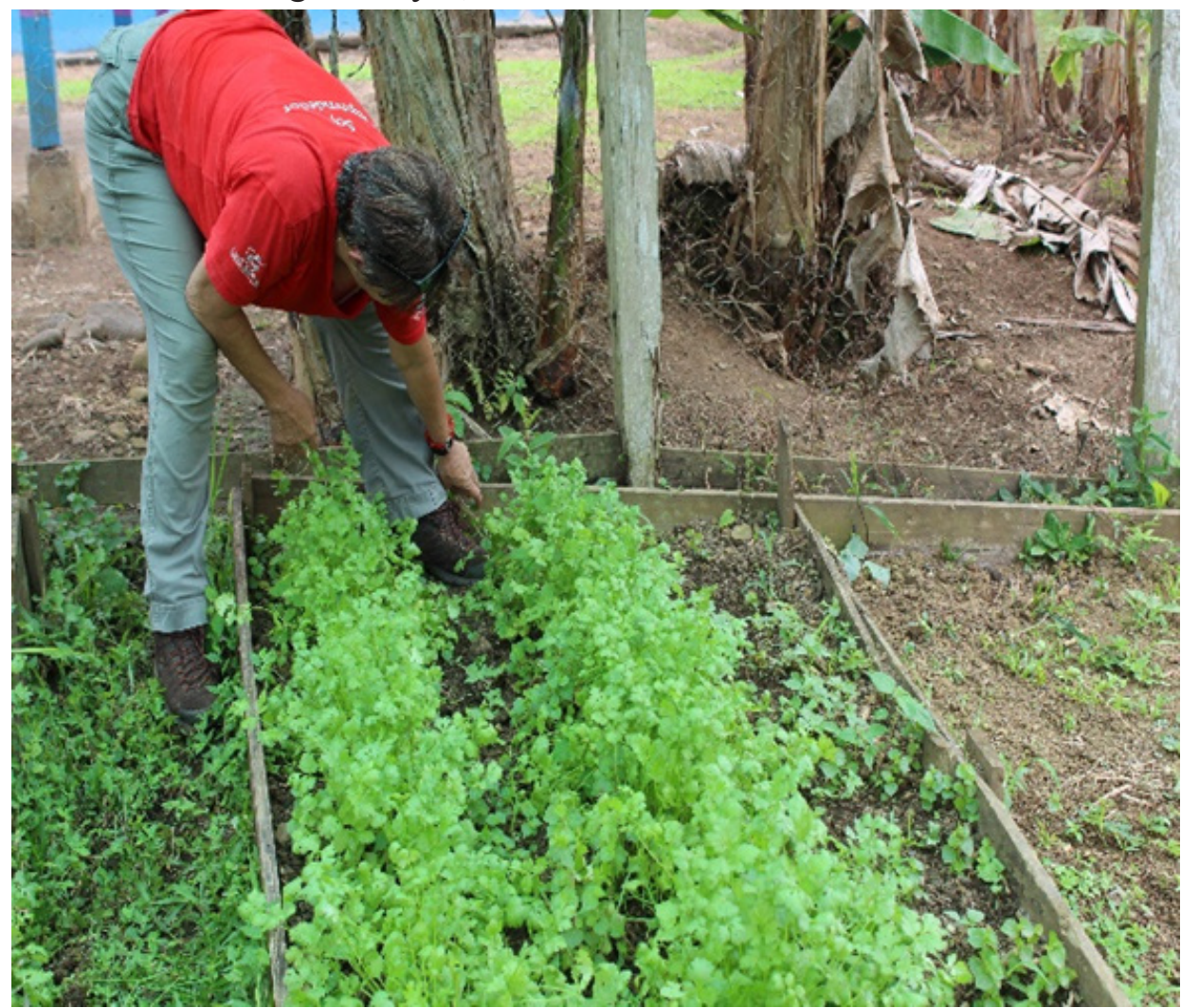

Fuente: Propia

- $\quad$ Año 3 Es en este año a raíz de los resultados obtenidos con la participación de los actores locales y de las instituciones públicas que se decide dar paso a que el proyecto y los actores involucrados tomen las riendas del mismo y sean capaces de seguir adelante ya con los nuevos conocimientos adquiridos pero bajo sus propios saberes autóctonos, también es importante resaltar que es en este año donde se involucra la comunidad de Los Ángeles Cábecar, a raíz de escuchar por tercero del proyecto y de manifestar tener una carencia que debe ser solventada. 
Sonia Lucía Montero-Herrera. Unidades Productivas Integrales, como un producto Turístico para el Desarrollo Rural Sustentable

Ilustración 4. UPI, comunal en la comunidad de Los Ángeles Cabecar, Territorio Indígena Tayni, Valle La Estrella Limón Costa Rica, 2018

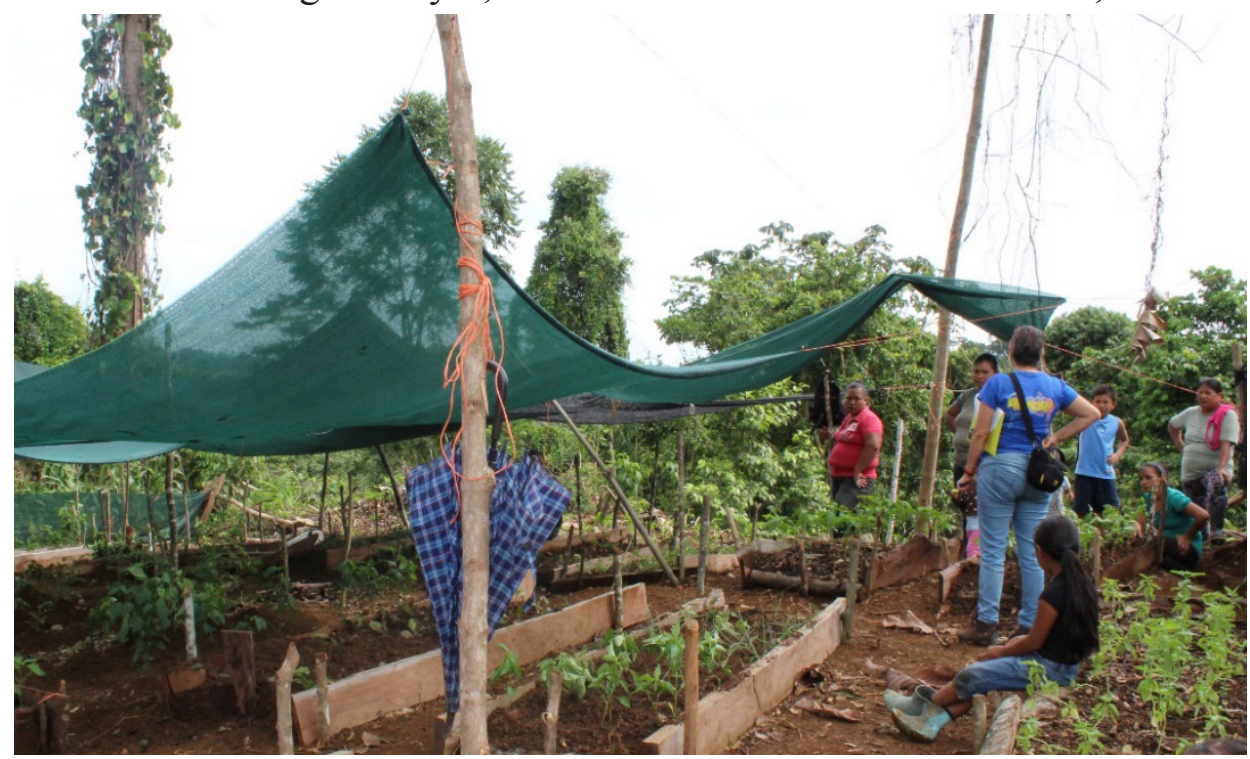

Fuente: Propia

- Año 4 y actual el inicio de este último año con las UPI establecidas y el reconocimiento de tales por las comunidades adyacentes se integran dos escuelas más la de Bellavista y Moi con las que ya se contaban dentro de los participantes y que son dirigidas bajo supervisión del equipo de la UNA pero con apoyo de los actores que ya tienen una experiencia dentro del proyecto. También dada la participación en talleres que se realizaron fuera del territorio a emprendimientos ya establecidos como lo es la Finca Educativa Agroecológica Don Juan en la Fortuna de San Carlos que surge un interés por parte principalmente de la Comunidad de los Ángeles de querer insertarse en sector turístico y convertirse en otra fuente alternativa de visitación turística para la zona. ES en este punto en el que se encuentra es este momento el proyecto como tal y que persigue como fin no solo crear otra fuente de ingresos sino que este territorio se considere un lugar atractivo que permita dar a conocer no solo sus riquezas naturales sino la riqueza cultural que posee como comunidad indígena del lugar. 


\section{Discusión}

El agro ecoturismo, con este nuevo modelo de las UPI surge como una actividad alternativa para el desarrollo de las comunidades rurales que puede mezclar no solo las actividades propias de una unidad productiva o finca sino que puede involucrar la recreación la gastronomía local la medicina tradicional y las artesanías. Es una nueva tendencia que está dirigida por los mismos propietarios con apoyo familiar o comunal y que es flexible a los encadenamientos productivos y comerciales donde el beneficio no es solo individual sino comunal. Cabe mencionar que no requiere de una gran inversión por parte de los interesados y que no altera la fuente de ingresos principal y que puede verse complementada son otros servicios como lo es el alojamiento la alimentación las visitas guiadas y el interactuar y compartir su cultura con los visitantes.

Es así como puede convertirse en una nueva fuente generadora de empleo en los espacios rurales, que puede realzar la cultura local reforzándola y enalteciéndola y que concuerde con la visión y cosmovisión de estos pueblos indígenas

\section{Conclusiones}

Existen diferentes resultados que se pueden concluir primero desde los diferentes ámbitos y escenarios posibles podemos decir que las UPI como actividad económica tiene una serie de beneficios tanto económicos, sociales, culturales y ambientales. Entre ellos podemos mencionar que la implementación de los talleres de apoyo por parte del equipo universitario brinda nueva herramientas para futuras gestiones hacia el desarrollo local, también fomenta la sensibilización hacia la conservación y protección del medio ambiente por medio de campañas dirigidas hacia este tema, y que pretende crear reflexiones futuras en el uso racional de los recursos naturales

En la parte económica como alternativa a las ya existentes brinda nuevas oportunidades de fuentes de empleo o de generación propia de ingresos que mejora las condiciones socioeconómicas de los involucrados en dicho proceso. También al producirse encadenamientos locales hace que todo el territorio se vea beneficiado y que las ganancias generadas no se conviertan en una fuga de capital sino que este pueda ser reinvertido en la misma zona donde se desarrolla el emprendimiento. 
Sonia Lucía Montero-Herrera. Unidades Productivas Integrales, como un producto Turístico para el Desarrollo Rural Sustentable

Por último y no menos importante es la parte cultural ya que el proyecto se basa en un respeto total por los conocimientos ancestrales y la cultura propia de los involucrados, poniéndola como una parte vital para el buen funcionamiento de las unidades productivas ya que la experiencia y puesta en práctica de estos conocimientos y que está generando un interés por parte de las generaciones futuras que lo habían dejado de lado. También como impacto positivo es que estas comunidades se están visibilizando en el mapa económico y turístico local, insertándolos como una nueva alternativa de visitación dentro de la oferta que tien2 la zona

\section{Referencias}

Arias, H. (2016). El ser humano y sostenibilidad. San José, Costa Rica: EUNED.

Borges, C. (2012). El Policultivo Indígena de Talamanca y la Conservación de la Naturaleza. San José, CR.: INBio. 221p.

Brenes, C. (2010). Comunidades Rurales: Criterios y herramientas para su diagnóstico. San José, Costa Rica: EUNED.

Crespo, P., D Rham, P., Gonzales, G., Iturralde, P., Jaramillo, B., Mancero, L., Moncada, M., Pérez, A. y Soria, C. (2007). Empoderamiento: Conceptos y orientaciones. Recuperado de www.asocam.org. (29 agosto 2017).

Gómez, C. (2017) Capítulo III. El desarrollo sostenible: conceptos básicos, alcance y criterios para su evaluación. En Desarrollo sostenible: origen del término y rasgos principales. UNESCO http://www.unesco.org/new/fileadmin/MULTIMEDIA/FIELD/Havana/pdf/Cap3.pdf Hernan, G. \& Dario, A. (2017). La conciencia ambiental en Costa Rica: Evolución, estado actual y retos futuros. San José, Costa Rica: Ministerio de Ambiente y Energia (MINAE).

Instituto Aguas calientes de la mujer,. (2017). Manual empoderamiento de las mujeres. Obtenido el 29 de agosto de 2017). http://www.aguascalientes.gob.mx/iam/LIBROS\%20PDF's/PUBLICACIONES\%20 ELECTRONICAS\%202007/Manual\%20Empoderamiento\%20 de $\% 201$ as $\% 20$ Mujeres.pdf.

Instituto Tecnológico de Costa Rica (2008) Atlas 2008. Elaborado por Chacón, N. 
MIDEPLAN (s.f.) http://mapas.mideplan.go.cr/regiones/ibina_regional/. Obtenido de http://mapas.mideplan.go.cr/regiones/ibina_regional/ (29 de agosto de 2017)

Morales, E. \& Condori, F. (2012). Diagnostico socio cultural y físico espacial de las comunidades ubicadas en el corredor fronterizo - cantón de Sarapiquí - Heredia. Recuperado el 29 de 8 de 2017 de https:// www.mivah.go.cr/Documentos/investigaciones_diagnosticos/diagnosticos planes intervencion/2012/SARAPIQUI_HEREDIA/DIAGNOSTICO_SARAPIQUI_FRONTERA.pdf.

Organización Mundial delTurismo(OMT).(2018). Glosario Básico. Organización Mundial del Turismo. Recuperado el 26 de abril del 2018. http:// media.unwto.org/es/content/entender-el-turismo-glosario-basico

Rojas, I. y Daly, C. (2016) Propuesta de un modelo para el desarrollo del turismo rural comunitario en la comunidad de los Ángeles en Cureña Sarapiquí Heredia, Costa Rica. En Revista Nuevo Humanismo, 4(2), 141-156 https://doi.org/10.15359/rnh.4-2.8

Roldán. C. A. (2008) Decimocuarto Informe Estado de La Nación en Desarrollo Humano Sostenible. Informe Final. Agricultura: tendencias recientes e implicaciones ambientales Un año de crisis climática y alimentaria. Pág. 3 Recuperado de https://estadonacion.or.cr/files/ biblioteca_virtual/014/Agricultura-Roldan-2008.pdf

Rojas, L. (2009). Decimoquinto Informe Estado de la Nación en Desarrollo Humano Sostenible. Informe Final Evolución e importancia del turismo en Costa Rica. https://estadonacion.or.cr/files/biblioteca_virtual/015/Rojas_2009.pdf 16-05-18

Secretaria Técnica de los Objetivos de Desarrollo Sostenible (ODS). (2016). Recuperado de www.ods.cr/recursos/publicaciones-y-documentos. (29 agosto 2017).

Universidad Nacional. (2015). Estatuto Organico. Heredia, Costa Rica: EUNA. 